\title{
Information, Physics, and Computation
}

\author{
Marc Mézard \\ Laboratoire de Physique Théorique et Modèles Statistiques, \\ CNRS, and Université Paris Sud \\ Andrea Montanari \\ Department of Electrical Engineering and Department of Statistics, \\ Stanford University, \\ and Laboratoire de Physique Théorique de l'ENS, Paris
}

\section{OXFORD \\ UNIVERSITY PRESS}




\section{Contents}

\section{PART I BACKGROUND}

1 Introduction to information theory 3

1.1 Random variables 3

1.2 Entropy 5

1.3 Sequences of random variables and their entropy rate 8

1.4 Correlated variables and mutual information 10

$\begin{array}{ll}1.5 & \text { Data compression } \\ 1.6\end{array}$

1.6 Data transmission 16

Notes $\quad 21$

$2 \quad$ Statistical physics and probability theory 23

2.1 The Boltzmann distribution $\quad 24$

2.2 Thermodynamic potentials 28

2.3 The fluctuation-dissipation relations 32

2.4 The thermodynamic limit 33

2.5 Ferromagnets and Ising models 35

2.6 The Ising spin glass 44

Notes $\quad 46$

$3 \quad$ Introduction to combinatorial optimization $\quad 47$

3.1 A first example: The minimum spanning tree 48

3.2 General definitions 51

3.3 More examples 51

3.4 Elements of the theory of computational complexity 54

$\begin{array}{ll}3.5 & \text { Optimization and statistical physics } \\ 3.6 & 60\end{array}$

3.6 Optimization and coding 61

Notes $\quad 62$

4 A probabilistic toolbox 65

4.1 Many random variables: A qualitative preview 65

4.2 Large deviations for independent variables 66

$\begin{array}{lll}4.3 & \text { Correlated variables } & 72\end{array}$

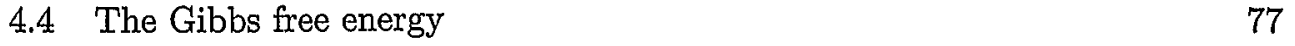

4.5 The Monte Carlo method 80

4.6 Simulated annealing 86

4.7 Appendix: A physicist's approach to Sanov's theorem 87

$\begin{array}{ll}\text { Notes } & 89\end{array}$ 


\section{PART ॥ INDEPENDENCE}

$5 \quad$ The random energy model 93

5.1 Definition of the model 93

5.2 Thermodynamics of the REM 94

5.3 The condensation phenomenon 100

5.4 A comment on quenched and annealed averages 101

5.5 The random subcube model 103

$\begin{array}{ll}\text { Notes } & 105\end{array}$

6 The random code ensemble 107

6.1 Code ensembles 107

6.2 The geometry of the random code ensemble 110

6.3 Communicating over a binary symmetric channel 112

6.4 Error-free communication with random codes 120

6.5 Geometry again: Sphere packing 123

6.6 Other random codes 126

6.7 A remark on coding theory and disordered systems 127

6.8 Appendix: Proof of Lemma 6.2 128

Notes 128

7 Number partitioning 131

7.1 A fair distribution into two groups? 131

$\begin{array}{ll}7.2 & \text { Algorithmic issues } \\ 732\end{array}$

7.3 Partition of a random list: Experiments 133

7.4 The random cost model 136

7.5 Partition of a random list: Rigorous results 140

Notes 143

8 Introduction to replica theory $\quad 145$

8.1 Replica solution of the random energy model 145

8.2 The fully connected $p$-spin glass model 155

8.3 Extreme value statistics and the REM 163

8.4 Appendix: Stability of the RS saddle point 166

$\begin{array}{ll}\text { Notes } & 169\end{array}$

PART III MODELS ON GRAPHS

9 Factor graphs and graph ensembles 173

$\begin{array}{lll}9.1 & \text { Factor graphs } & 173\end{array}$

$\begin{array}{ll}\text { 9.2 Ensembles of factor graphs: Definitions } & 180\end{array}$

9.3 Random factor graphs: Basic properties 182

$\begin{array}{ll}\text { 9.4 Random factor graphs: The giant component } & 187\end{array}$

9.5 The locally tree-like structure of random graphs 191

Notes 194

$\begin{array}{ll}10 \text { Satisfiability } & 197\end{array}$

$\begin{array}{ll}10.1 \text { The satisfiability problem } & 197\end{array}$ 
10.2 Algorithms 199

10.3 Random $K$-satisfiability ensembles 206

10.4 Random 2-SAT 209

10.5 The phase transition in random $K(\geq 3)$-SAT 209

$\begin{array}{ll}\text { Notes } & 217\end{array}$

11 Low-density parity-check codes 219

$\begin{array}{ll}11.1 \text { Definitions } & 220\end{array}$

11.2 The geometry of the codebook 222

11.3 LDPC codes for the binary symmetric channel 231

11.4 A simple decoder: Bit flipping $\quad 236$

$\begin{array}{ll}\text { Notes } & 239\end{array}$

12 Spin glasses $\quad 241$

12.1 Spin glasses and factor graphs 241

12.2 Spin glasses: Constraints and frustration 245

$\begin{array}{ll}12.3 \text { What is a glass phase? } & 250\end{array}$

12.4 An example: The phase diagram of the SK model 262

Notes 265

13 Bridges: Inference and the Monte Carlo method 267

13.1 Statistical inference 268

13.2 The Monte Carlo method: Inference via sampling $\quad 272$

$\begin{array}{ll}13.3 \text { Free-energy barriers } & 281\end{array}$

$\begin{array}{ll}\text { Notes } & 287\end{array}$

\section{PART IV SHORT-RANGE CORRELATIONS}

14 Belief propagation $\quad 291$

14.1 Two examples $\quad 292$

14.2 Belief propagation on tree graphs 296

14.3 Optimization: Max-product and min-sum 305

$\begin{array}{ll}14.4 \text { Loopy BP } & 310\end{array}$

14.5 General message-passing algorithms 316

$\begin{array}{lr}14.6 & \text { Probabilistic analysis } \\ & 317\end{array}$

Notes $\quad 325$

15 Decoding with belief propagation 327

15.1 BP decoding: The algorithm 327

$\begin{array}{lr}15.2 \text { Analysis: Density evolution } & 329\end{array}$

15.3 BP decoding for an erasure channel $\quad 342$

15.4 The Bethe free energy and MAP decoding 347

Notes $\quad 352$

16 The assignment problem 355

16.1 The assignment problem and random assignment ensembles 356

16.2 Message passing and its probabilistic analysis 357

16.3 A polynomial message-passing algorithm 366 
xii Contents

$\begin{array}{ll}\text { 16.4 Combinatorial results } & 371\end{array}$

16.5 An exercise: Multi-index assignment $\quad 376$

Notes $\quad 378$

17 Ising models on random graphs 381

17.1 The BP equations for Ising spins $\quad 381$

$\begin{array}{ll}17.2 & \text { RS cavity analysis } \\ 17.3 & 384\end{array}$

17.3 Ferromagnetic model 386

$\begin{array}{ll}17.4 \text { Spin glass models } & 391\end{array}$

$\begin{array}{ll}\text { Notes } & 399\end{array}$

PART V LONG-RANGE CORRELATIONS

18 Linear equations with Boolean variables 403

18.1 Definitions and general remarks 404

$\begin{array}{ll}18.2 \text { Belief propagation } & 409\end{array}$

18.3 Core percolation and BP 412

18.4 The SAT-UNSAT threshold in random XORSAT 415

18.5 The Hard-SAT phase: Clusters of solutions 421

18.6 An alternative approach: The cavity method 422

$\begin{array}{ll}\text { Notes } & 427\end{array}$

19 The 1RSB cavity method 429

19.1 Beyond BP: Many states $\quad 430$

19.2 The 1RSB cavity equations $\quad 434$

19.3 A first application: XORSAT 444

19.4 The special value $x=1 \quad 449$

19.5 Survey propagation $\quad 453$

19.6 The nature of 1RSB phases 459

19.7 Appendix: The SP $(y)$ equations for XORSAT 463

Notes $\quad 465$

20 Random K-satisfiability $\quad 467$

20.1 Belief propagation and the replica-symmetric analysis 468

20.2 Survey propagation and the 1RSB phase $\quad 474$

20.3 Some ideas about the full phase diagram $\quad 485$

20.4 An exercise: Colouring random graphs 488

Notes $\quad 491$

21 Glassy states in coding theory 493

21.1 Local search algorithms and metastable states 493

21.2 The binary erasure channel $\quad 500$

21.3 General binary memoryless symmetric channels 506

21.4 Metastable states and near-codewords $\quad 513$

Notes $\quad 515$

22 An ongoing story 517

22.1 Gibbs measures and long-range correlations $\quad 518$ 
22.2 Higher levels of replica symmetry breaking

22.3 Phase structure and the behaviour of algorithms $\quad 535$

Notes

538

Appendix A Symbols and notation $\quad 541$

A.1 Equivalence relations $\quad 541$

A.2 Orders of growth $\quad 542$

A.3 Combinatorics and probability 543

A.4 Summary of mathematical notation $\quad 544$

A.5 Information theory $\quad 545$

A.6 Factor graphs $\quad 545$

A.7 Cavity and message-passing methods $\quad 545$

$\begin{array}{ll}\text { References } & 547\end{array}$

$\begin{array}{ll}\text { Index } & 565\end{array}$ 